\title{
IscR Regulation of Capsular Polysaccharide Biosynthesis and Iron-Acquisition Systems in Klebsiella pneumoniae CG43
}

\author{
Chien-Chen $\mathrm{Wu}^{19}$, Chien-Kuo Wang ${ }^{29}$, Yu-Ching Chen ${ }^{39}$, Tien-Huang Lin ${ }^{4,5}$, Tzyy-Rong Jinn ${ }^{5}$, \\ Ching-Ting Lin $^{5 *}$
}

1 Institute of Biochemistry and Molecular Biology, National Yang-Ming University, Taipei, Taiwan, Republic of China, 2 Department of Biotechnology, Asia University, Taichung, Taiwan, Republic of China, 3 Department of Biomedical Informatics, Asia University, Taichung, Taiwan, Republic of China, 4 Division of Urology, Taichung Tzu Chi Hospital, The Buddhist Tzu Chi Medical Foundation, Taichung, Taiwan, Republic of China, $\mathbf{5}$ Graduate Institute of Chinese Medicine, School of Chinese Medicine, China Medical University, Taichung, Taiwan, Republic of China

\begin{abstract}
IscR, an Fe-S cluster-containing transcriptional factor, regulates genes involved in various cellular processes. In response to environmental stimuli such as oxidative stress and iron levels, IscR switches between its holo and apo forms to regulate various targets. IscR binding sequences are classified into two types: the type 1 IscR box that is specific for holo-IscR binding, and the type 2 IscR box that binds holo- and apo-IscR. Studying Klebsiella pneumoniae CG43S3, we have previously shown that iron availability regulates capsular polysaccharide (CPS) biosynthesis and iron-acquisition systems. The present study investigated whether IsCR is involved in this regulation. Compared with that in CG43S3, the amount of CPS was decreased in AP001 ( $\triangle i s C R$ ) or AP002 (iscR $R_{3 C A}$ ), a CG43S3-derived strain expressing mutated IscR mimicked apo-IscR, suggesting that only holo-IscR activates CPS biosynthesis. Furthermore, a promoter-reporter assay verified that the transcription of $c p s$ genes was reduced in AP001 and AP002. Purified IscR::His ${ }_{6}$, but not IscR ${ }_{3 C A}:: \mathrm{His}_{6}$, was also found to bind the predicted type 1 IscR box specifically in the cps promoter. Furthermore, reduced siderophore production was observed in AP004 ( $\triangle$ fur- $\triangle i s c R)$ but not in AP005 ( $\Delta$ fur-iscR $\left.R_{3 C A}\right)$, implying that apo-IscR activates iron acquisition. Compared with those in AP004, mRNA levels of three putative iron acquisition systems ( $f h u$, iuc, and sit) were increased in AP005, and both purified IscR::His ${ }_{6}$ and $I s c R_{3 c A}:: H_{i s}$ bound the predicted type 2 IscR box in the fhuA, iucA, and sitA promoters, whereas IscR ${ }_{3 c A}:: H_{i s} \operatorname{displayed~a~}$ lower affinity. Finally, we analyzed the effect of external iron levels on isc $R$ expression. The transcription of isc $R$ was increased under iron-depleted conditions as well as in AP001 and AP002, suggesting an auto-repression exerted by apoIscR. Our results show that in K. pneumoniae, IscR plays a dual role in the regulation of CPS biosynthesis and iron-acquisition systems in response to environmental iron availability.
\end{abstract}

Citation: Wu C-C, Wang C-K, Chen Y-C, Lin T-H, Jinn T-R, et al. (2014) IscR Regulation of Capsular Polysaccharide Biosynthesis and Iron-Acquisition Systems in Klebsiella pneumoniae CG43. PLoS ONE 9(9): e107812. doi:10.1371/journal.pone.0107812

Editor: José A. Bengoechea, Queen's University Belfast, United Kingdom

Received March 3, 2014; Accepted August 17, 2014; Published September 19, 2014

Copyright: $\odot 2014$ Wu et al. This is an open-access article distributed under the terms of the Creative Commons Attribution License, which permits unrestricted use, distribution, and reproduction in any medium, provided the original author and source are credited.

Funding: The work is supported by the grants from National Science Council (NSC 99-2320-B-039-002-MY3 and NSC 102-2320-B-039-042-), China Medical University (CMU99-ASIA-07 and CMU100-ASIA-12), and Taichung Tzuchi Hospital, The Buddhist Tzu Chi Medical Foundation (TTCRD-10218). The funders had no role in study design, data collection and analysis, decision to publish, or preparation of the manuscript.

Competing Interests: The authors have declared that no competing interests exist.

* Email: gingting@mail.cmu.edu.tw

9 These authors contributed equally to this work.

\section{Introduction}

Klebsiella pneumoniae is a rod-shaped, gram-negative bacterium that causes community-acquired diseases including pneumonia, bacteremia, septicemia, and urinary and respiratory tract infections that occurr particularly in immune-compromised patients [1]. In Asian countries, especially in Taiwan and Korea, $K$. pneumoniae is the predominant pathogen responsible for pyogenic liver abscesses in diabetic patients [2,3,4]. Among the virulence factors identified in $K$. pneumoniae, capsular polysaccharide (CPS) is considered the major determinant for $K$. pneumoniae infections. Pyogenic liver abscess isolates often carry heavy CPS loads that protect bacteria from phagocytosis and from being killed by serum factors $[5,6]$. Apart from its anti-phagocytic function, Klebsiella CPS also promotes bacterial colonization and biofilm formation at infection sites [7,8,9].
Our previous studies have demonstrated that CPS biosynthesis in $K$. pneumoniae is repressed in iron-replete conditions, and this regulation is controlled by an iron uptake regulator (Fur) [10]. Under iron-replete conditions, dimeric Fur in complex with $\mathrm{Fe}(\mathrm{II})$ indirectly activates $K$. pneumoniae CPS biosynthesis through transcriptional factors RmpA and RcsA and a small non-coding RNA, RyhB [10,11]. The transcription of cps genes is directly regulated by $\mathrm{RmpA}$ and $\mathrm{R} c \mathrm{~A}$ but appears to be indirectly regulated by RyhB. These findings indicate that environmental iron availability influences $K$. pneumoniae CPS biosynthesis through multiple regulators.

To maintain iron homeostasis, Fur acts as a master regulator to control iron transport, storage, and metabolism in many gramnegative bacteria including $K$. pneumoniae [11-13]. We have previously reported that Fur directly represses at least six of the 
eight iron acquisition systems in K. pneumoniae CG43S3 [10]. In addition to Fur, the transcriptional regulator IscR plays a crucial role in iron metabolism. IscR regulates the biosynthesis of Fe-S clusters, which are key cofactors of proteins intervening in various cellular processes in bacteria [12,13]. Fe-S clusters can be generally classified into two types, rhombic [2Fe-2S] and cubic $[4 \mathrm{Fe}-4 \mathrm{~S}]$, which have either ferrous $\left(\mathrm{Fe}^{2+}\right)$ or ferric $\left(\mathrm{Fe}^{3+}\right)$ iron and sulphide $\left(\mathrm{S}^{2-}\right)[14,15]$. IscR is itself a [2Fe-2S] cluster-containing protein encoded by the first gene of the iscRSUA operon. The switch between the [2Fe-2S] holo and apo forms of IscR is believed to be influenced by environmental conditions such as oxidative and nitric oxide stress and cellular iron levels $[13,16,17,18]$. Moreover, holo- and apo-IscR have been shown to regulate different target genes, suggesting that the presence of the $[2 \mathrm{Fe}-2 \mathrm{~S}]$ cluster affects the regulatory specificity of IscR $[18,19,20,21]$.

Transcriptomic analysis has identified 40 genes in 20 predicted operons, which are regulated by IscR under aerobic and anaerobic conditions in Escherichia coli [19]. This analysis has also revealed two classes of IscR binding sites (IscR boxes). Type 1 IscR box consists of a 25-bp sequence interacted with holo-IscR, whereas type 2 IscR box consists of a 26-bp sequence interacted with apoIscR [19]. Furthermore, a detailed analysis of the type 2 IscR box has verified an IscR binding motif for both holo and apo-IscR binding [21].

In this study, we investigated whether IscR participates in the regulation of CPS biosynthesis and the expression of iron acquisition systems in $K$. pneumoniae. We also analysed the expression of $i s c R$ in response to various iron levels.

\section{Results}

IscR activates $K$. pneumoniae CPS biosynthesis in an Fe-S cluster-dependent manner

To study whether IscR regulates $K$. pneumoniae CPS biosynthesis, we determined the amounts of K2 CPS in CG43S3 (wild type [WT]) and AP001 ( $\Delta i s c R)$ strains. Compared with the WT, AP001 produced significantly lower amounts of CPS (Fig. 1A), suggesting that IscR activates the biosynthesis of CPS. In $K$. pneumoniae, IscR contains three highly conserved cysteine residues (C92, C98, and C104 in E. coli IscR) which are thought to coordinate the [2Fe-2S] cluster [20].

To investigate the role of the $[2 \mathrm{Fe}-2 \mathrm{~S}]$ cluster in IscR regulation of CPS biosynthesis, we created an isc $R$ mutant AP002 (isc $R_{3 \mathrm{CA}}$ ) by replacing the three cysteines with alanines and tested whether this mutant, which is predicted to encode an IscR lacking an Fe-S cluster, affected CPS biosynthesis. As shown in Fig. 1A, we found that the amount of CPS decreased in the AP002 strain compared with that in the WT, indicating that the regulation of $\mathrm{IscR}$ required the $[2 \mathrm{Fe}-2 \mathrm{~S}]$ cluster. Moreover, isc $R$ and $i s c R_{3 \mathrm{CA}}$ were respectively cloned into pACYC184, to yield pIscR and $\mathrm{pIscR}_{3 \mathrm{CA}}$, for complementation analysis. Compared with AP001 [pACYC184], AP001 [pIscR] produced a significantly higher amount of CPS, whereas the introduction of $\mathrm{pIscR}_{3 \mathrm{CA}}$ into the AP001 strain did not change the CPS amount (Fig. 1B). These results confirmed that IscR has a positive role in the regulation of CPS biosynthesis and that the presence of the [2Fe-2S] cluster of IscR is essential for this regulation. On the other hand, the CPS amount appeared to obviously increased in AP001 [pIscR], compared with that in WT [pACYC184] (Fig. 1B), which may result from multicopy plasmids are used for complementation. Therefore, we also used single copy constructs to complement the iscR-deletion (Methods $\mathrm{S} 1$ ), and the result showed that the expression of $i s c R$, but not isc $R_{3 \mathrm{CA}}$, could restore the CPS biosynthesis (Fig. $\mathrm{S} 1$ ).
The K2 cps gene cluster of $K$. pneumoniae contains 19 open reading frames (ORFs) organised into 3 transcription unitsnamely, orf 1-2, orf3-15, and orf16-17 [22]. To determine the role of IscR in regulating $c p s$ transcription, we used the reporter

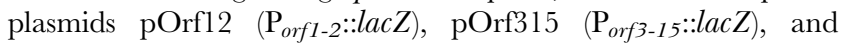

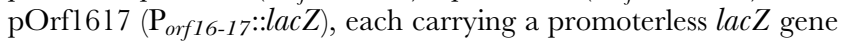
transcriptionally fused to the putative promoter region of the $\mathrm{K} 2$ cps gene cluster [23], to transform the $K$. pneumoniae strains

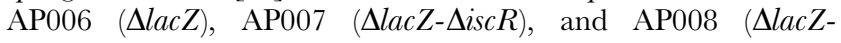
$\left.i s c R_{3 \mathrm{CA}}\right)$. The measurements shown in Fig. $1 \mathrm{C}$ reveal that the promoter activity of orf1-2 and orf16-17 in AP007 and AP008 was lower than that in AP006 $(P<0.01)$. Additionally, the promoter activity of orf3-15 was apparently decreased in AP008 but slightly decreased in AP007 compared with that in AP006. These results indicated that $\mathrm{Isc} R$ activates the transcription of $c p s$ genes in an Fe-S cluster-dependent manner.

\section{IscR directly binds the promoter of galF}

For further investigation of the mechanism of IscR regulation on $c p s$ transcription, the sequence of the putative IscR binding site was manually analysed in the three promoter regions of the $\mathrm{K} 2 c p s$ gene cluster. As shown in Fig. 2A, we found a putative type 1 IscR box with $52 \%$ (13/25 bp) homology to the consensus sequence located between $-173 \mathrm{bp}$ and $-197 \mathrm{bp}$ relative to the translational start codon of galF (orf1 in the $\mathrm{K} 2$ cps gene cluster). In addition, the putative IscR binding sequence in $\mathrm{P}_{\text {galF }}$ was highly homologous to the IscR-binding motif (5'-AxxxCGxxAxxxxxxxTAxGGxxxT-3') reported by Nesbit et al. [21]. However, no typical IscR binding site was found in the upstream sequence of $w z i$ (orf3 in the $\mathrm{K} 2$ cps gene cluster) or manC (orf16 in the $\mathrm{K} 2$ cps gene cluster), suggesting that $\mathrm{IscR}$ indirectly regulates the promoter activities of orf $3-15$ and orf 16-17, which remains to be studied. On the other hand, we hypothesised that IscR binds directly to the promoter region of galF to activate gene transcription, and we confirmed this by performing an electrophoretic mobility shift assay (EMSA). As shown in the upper panel of Fig. 2B, purified recombinant IscR::His 6 protein was able to bind PgalF-1 but not PgalF-2, in which the region containing the putative IscR binding site was deleted. In addition, compared with that of IscR::His 6 , the recombinant [2Fe-2S] clusterless IscR $_{3 \mathrm{CA}}::-$ $\mathrm{His}_{6}$ had reduced PgalF-1 binding activity. Furthermore, no obvious interaction between the recombinant IscR proteins and PgalF-1*, the galF promoter lacking only the 25-bp predicted IscR box, was found (the lower panel of Fig. 2B). Besides, PgalF-1 and PgalF-1* DNA showed a slightly different mobility in the gel. These results suggested a direct interaction between IscR and the galF promoter and that the [2Fe-2S] cluster of IscR plays a crucial role in this interaction. On the contrary, we also analysed whether recombinant IscR::His ${ }_{6}$ could bind the promoter regions of $w z i$ and manC. As expected, EMSA showed no obvious DNA-protein complex (data not shown).

\section{Effect of IscR on normal human serum resistance}

Because CPS acts as a protectant for $K$. pneumoniae against serum factors, we hypothesize that through modulation of CPS levels, IscR affects the ability of $K$. pneumoniae to resist the bactericidal effects of serum. To test this hypothesis, we treated $K$. pneumoniae strains with $75 \%$ normal human serum and determined their survival rates. Compared with the WT, the AP001 and AP002 strains had a slightly reduced survival rate (Fig. 3A), implying a positive role for [2Fe-2S]-IscR in the serum resistance of $K$. pneumoniae. To confirm this result further, we performed a complementation study. As shown in Fig. 3B, the introduction of $\mathrm{pIscR}$, but not pACYC184 or $\mathrm{pIscR}_{3 \mathrm{CA}}$, into the 
A

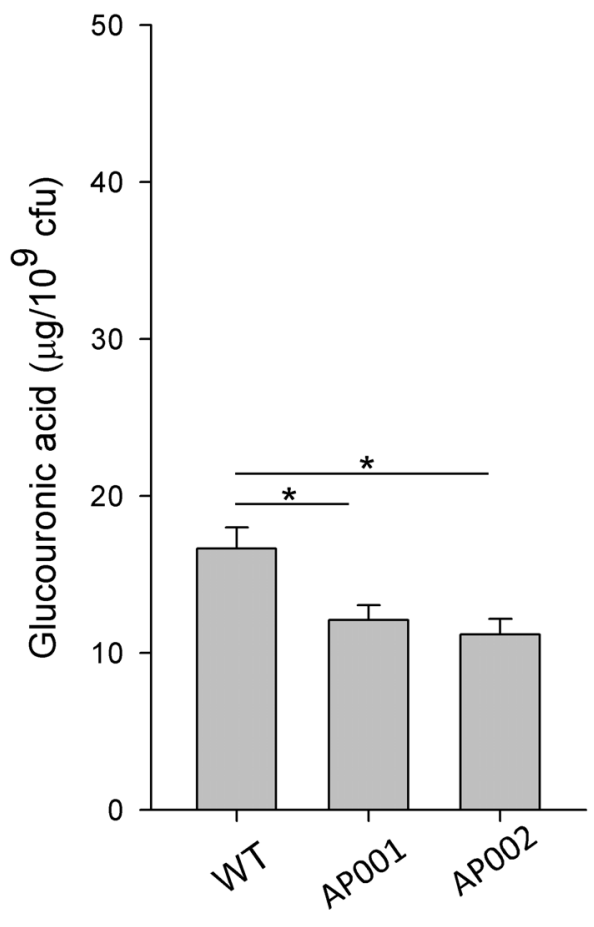

C



B

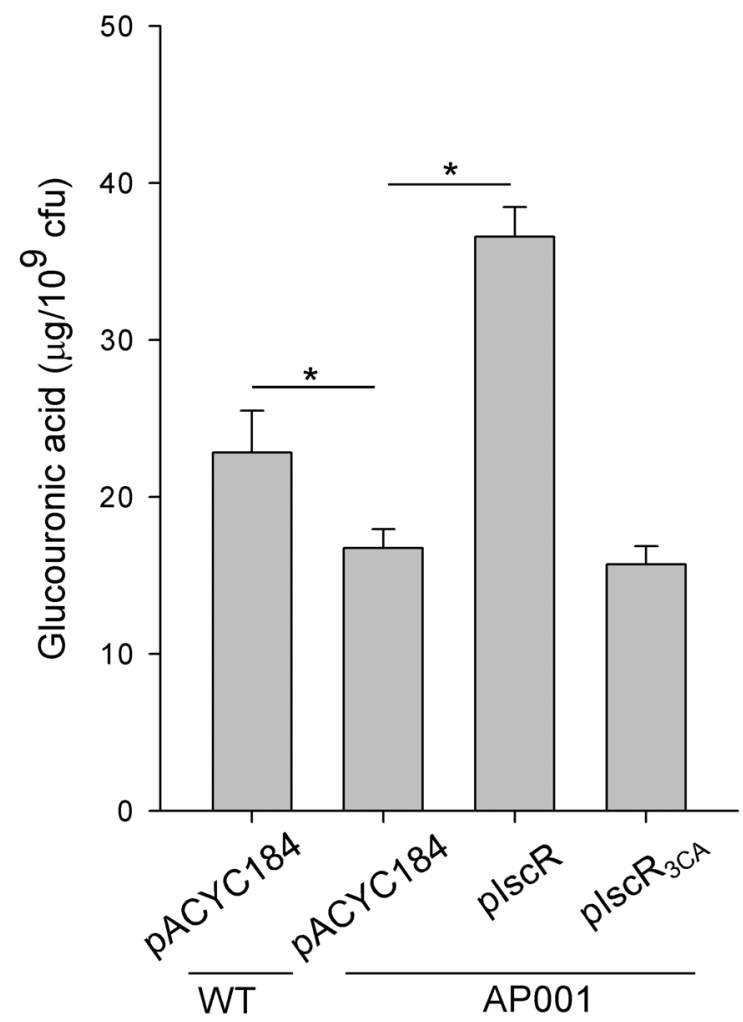

Figure 1. Holo-IscR positively regulates the biosynthesis of CPS. (A) CPS levels of the WT, AP001, and AP002 strains grown in LB broth. (B) CPS levels in WT carrying pACYC184 and AP001 carrying PACYC184, plscR, or plscR 3 CA were determined in LB. Bacterial glucuronic acid content was determined after $16 \mathrm{~h}$ of growth. (C) $\beta$-Galactosidase activities of $K$. pneumoniae AP006 and isogenic strains (AP007 and AP008) carrying the reporter


bars indicate standard deviations. ${ }^{*} P<0.01$ compared with the indicated groups.

doi:10.1371/journal.pone.0107812.g001

AP001 strain increased the bacterial survival rate, to a similar level compared with that of WT [pACYC184], after serum treatment. These results supported the hypothesis that [2Fe-2S]-IscR activates the expression of CPS to increase $K$. pneumoniae resistance to normal human serum.
IscR has a regulatory role in iron acquisition systems

In E. coli, both Fur and IscR play important roles in the maintenance of cellular iron homeostasis [12,13]. To analyse whether IscR regulates iron acquisition in $K$. pneumoniae, we performed a chrome azurol $\mathrm{S}$ (CAS) assay to assess siderophore 
A
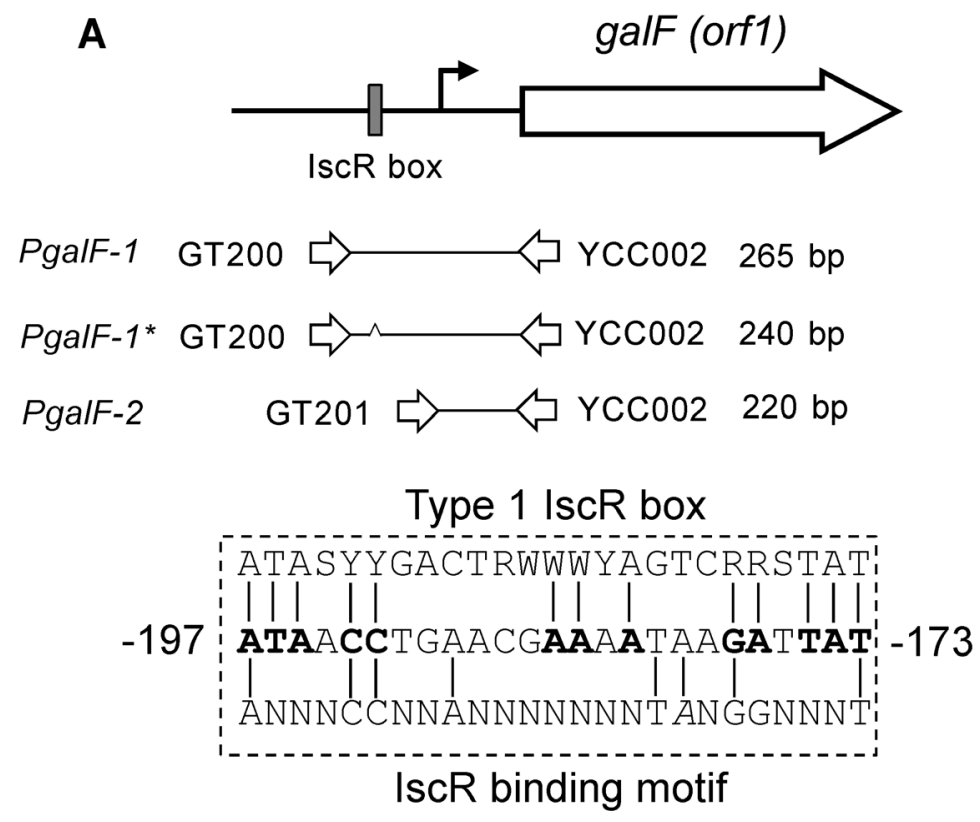

B

\begin{tabular}{|c|c|c|c|c|c|}
\hline \multicolumn{5}{|c|}{ PgalF-1 } & \multirow{2}{*}{$\begin{array}{r}\text { PgalF-2 } \\
\text { IscR::His }_{6}\end{array}$} \\
\hline & & ${ }_{3 \mathrm{CA}}: \because \mathrm{His}_{6}$ & & & \\
\hline$[\mathrm{nM}] \quad 0$ & 2 & $\overline{10}$ & 2 & $\overline{10}$ & $\begin{array}{lll}0 & 4 & 10\end{array}$ \\
\hline
\end{tabular}
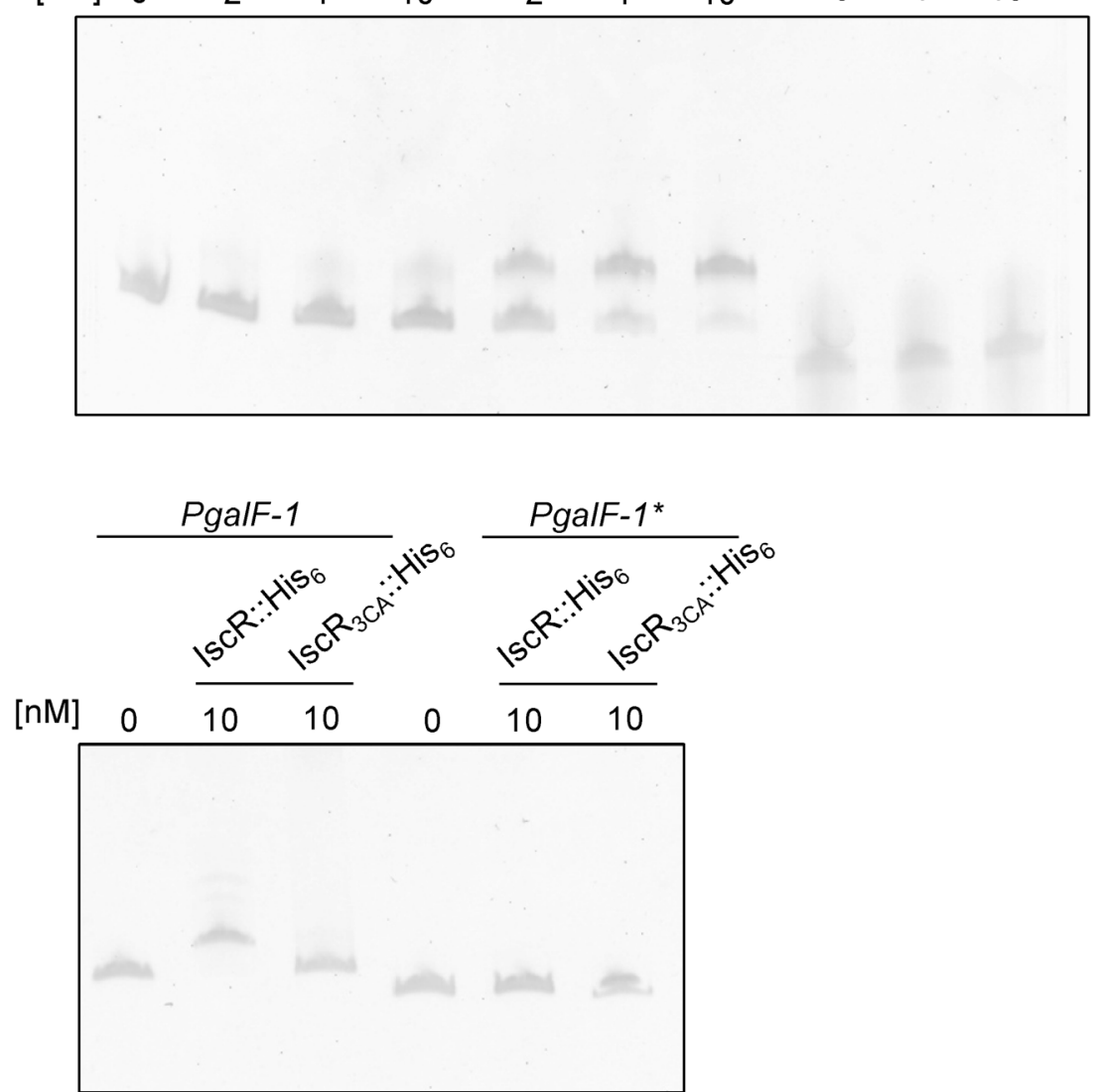

Figure 2. IscR binds directly to $\mathbf{P}_{\text {galF. }}$ (A) Diagrammatic representation of the galF loci. The primer sets used in PCR amplification of the DNA probes are indicated, and the numbers denote the DNA amplified length. DNA probes are listed on the left. The box in grey indicates the predicted type $1 \mathrm{IscR}$ box. The dashed box indicates the DNA sequence alignment among the predicted type $1 \mathrm{IscR}$ box, the IscR binding motif, and the putative IscR binding sequence in $\mathrm{P}_{\text {galf }}$, and the numbers denote the positions relative to the translational start site. Deletion of the predicted IscR 
box on PgalF-1* is indicated by a caret. (B) EMSA of IscR recombinant proteins and various DNA fragments of the upstream regions of galF. Different concentrations of purified IscR::His ${ }_{6}$ or $I_{S C R} \mathrm{R}_{3 \mathrm{~A}}:: \mathrm{His}_{6}$ were incubated with $5 \mathrm{ng}$ of DNA fragments, as indicated in the margin. After incubation at room temperature for $30 \mathrm{~min}$, the mixtures were resolved on a 5\% non-denaturing polyacrylamide gel. The gel was stained with SYBR Green I dye and photographed.

doi:10.1371/journal.pone.0107812.g002

secretion in K. pneumoniae strains. As shown in Fig. 4A, no apparent siderophore secretion was detected in the WT, AP001, or AP002 strains. Moreover, as in our previous report [10], deletion of fur clearly increased halo formation on the CAS plate. However, the halo was reduced in the AP003 ( $\Delta$ fur) strain background by the further deletion of $i s c R$, indicating the positive role of IscR in iron acquisition. Furthermore, no obvious difference in siderophore secretion was found between the AP003 and AP005 ( $\Delta$ fur- isc $R_{3 \mathrm{CA}}$ ) strains, suggesting that the IscR regulation of iron acquisition does not require the [2Fe-2S] cluster.

To verify whether apo-IscR activates iron acquisition, we introduced pACYC184, pIscR, or pIscR $3 \mathrm{CA}$ into the AP004 ( $\Delta$ fur$\Delta i s c R)$ strain and performed a CAS assay. As shown in Fig. 4B, the introduction of both $\mathrm{pIscR}$ and $\mathrm{pIscR}_{3 \mathrm{CA}}$ increased the halo phenotype on the CAS plate compared with that of the vector-

A

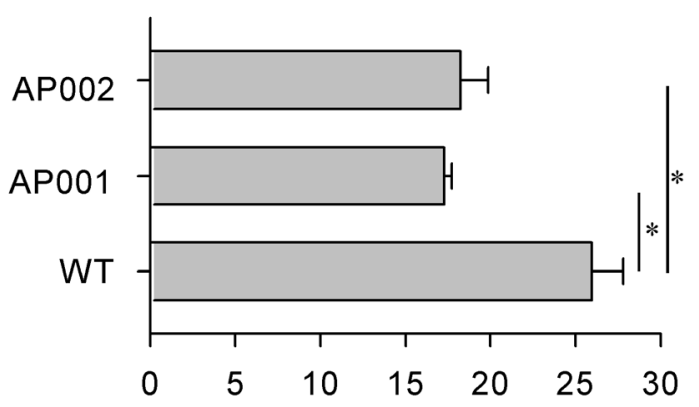

B

Survival rate $(\%)$



Survival rate $(\%)$

Figure 3. Deletion effect of iscR on $K$. pneumoniae susceptibility to normal human serum. The susceptibility to normal human serum of each bacterial mutant (A) and the complement strains (B) indicated in the margin was determined. Bacterial serum resistance was determined using log-phase cultures grown in LB medium. ${ }^{*} P<0.01$ compared with the indicated groups.

doi:10.1371/journal.pone.0107812.g003 only control. These results confirmed that IscR activates siderophore secretion in a $[2 \mathrm{Fe}-2 \mathrm{~S}]$ cluster-independent manner.

To further investigate the regulatory effect of IscR on iron acquisition, we used quantitative reverse transcription polymerase chain reaction (qRT-PCR) to measure the expression of genes corresponding to the eight putative iron acquisition systems in the indicated $K$. pneumoniae strains. As shown in Table 1, messenger RNA (mRNA) levels of genes (fhuA, iucA, and sitA) corresponding to three iron acquisition systems were increased more than 2-fold in the AP005 strain as compared with that in the AP004 strain. To further confirm this result, pACYC184, $\mathrm{pIscR}_{3 \mathrm{CA}}$, or pIscR were respectively introduced into the $\mathrm{AP004}$ strain, to avoid the effects of Fur, and the transcription of fhuA, iucA, and sitA were measured. The introduction of $\mathrm{pIscR}_{3 \mathrm{CA}}$ into AP004 apparently increased the transcription of fhuA, iucA, and sitA compared with that in the AP004 strain carrying pACYC184 only (Table 1). These results implied that apo-IscR activates the transcription of $f h u$, iuc, and sit to increase iron acquisition in K. pneumoniae. Besides, the introduction of pIscR into AP004 also slightly increased transcription of fhu, iuc, and sit (Table 1).

$\mathrm{IscR}_{3 C A}$ directly binds the promoter region of fhuA, iucA, and sitA

Apo-IscR has been demonstrated to bind the type 2 IscR box in IscR-regulated promoter sequences directly in E. coli [19]. Analysis of the promoter regions of $f h u A, i u c A$, and sitA revealed consensus sequences of the $E$. coli type 2 IscR box. As shown in Fig. 5A, the predicted type $2 \mathrm{IscR}$ boxes are located at -154 to -130 relative to the translation start site of fhuA and -67 to -43 relative to the translation start site of $i u c A$. The predicted type 2 IscR boxes in $\mathrm{P}_{f h u A}$ and $\mathrm{P}_{\text {iuc }}$ have $50 \%$ (13/26 bp) and 46\% (12/ $26 \mathrm{bp})$ homology, respectively, with the consensus sequence. In addition, two putative type 2 IscR boxes (R1 and R2) located at -112 to -87 and at -53 to -28 relative to the translation start site of sitA were found in $\mathrm{P}_{\text {sit }}$. The $\mathrm{R} 1$ and $\mathrm{R} 2$ sites contain $50 \%$ $(13 / 26 \mathrm{bp})$ and $61.5 \%$ (16/26 bp) homology, respectively, with the consensus sequence.

To verify whether apo-IscR binds to these predicted type 2 IscR boxes, we performed an EMSA. As shown in Fig. 5B-D, both the purified IscR::His ${ }_{6}$ and IscR $_{3 \mathrm{CA}}:: \mathrm{His}_{6}$ were able to bind with the promoter regions of $f h u A$, iucA, and $s i t A$, and $\mathrm{IscR}_{\mathrm{s}}: \mathrm{His}_{6}$ appeared to contain higher binding activities. Furthermore, $\mathrm{IscR}_{3 \mathrm{CA}}: \because \mathrm{His}_{6}$ did not bind PfhuA-2 and PiucA-2, which lacked a region containing a putative IscR box (Fig. $6 \mathrm{~B}-\mathrm{C}$ ). We also found that $\mathrm{IscR}_{3 \mathrm{CA}}:: \mathrm{His}_{6}$ did not bind PiucA-2, which contained the $\mathrm{R} 1$ site but not the R2 site (Fig. 6D). To further confirm the importance of these predicted IscR boxes, recombinant IscR proteins were respectively interacted with these promoters lacking only the 26-bp predicted type $2 \operatorname{IscR}$ box $\left(P f h u A-1^{*}, P i u c A-1^{*}\right.$, and PiucA-1*), and no obvious interaction was found. These results suggested that apo-IscR interacts directly with the promoters of $f h u A$, iucA, and sitA via the predicted type 2 IscR boxes.

Regulatory control of isc $R$ transcription in K. pneumoniae

To analyse whether environmental iron availability affects $K$. pneumoniae isc $R$ expression, we grew AP006 in Luria-Bertani (LB) broth supplemented with increasing amounts of the iron chelator 2, 2-dipyridyl (Dip) and monitored the promoter activity of $i s c R$ 
A

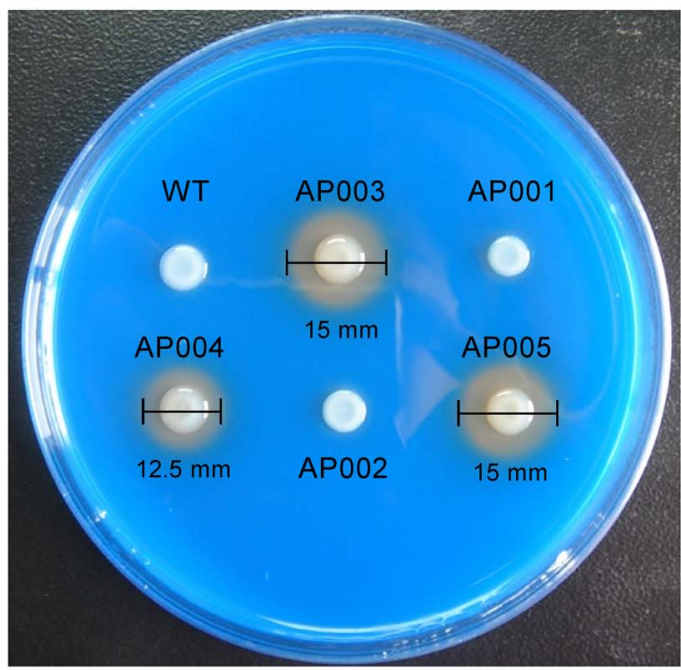

B

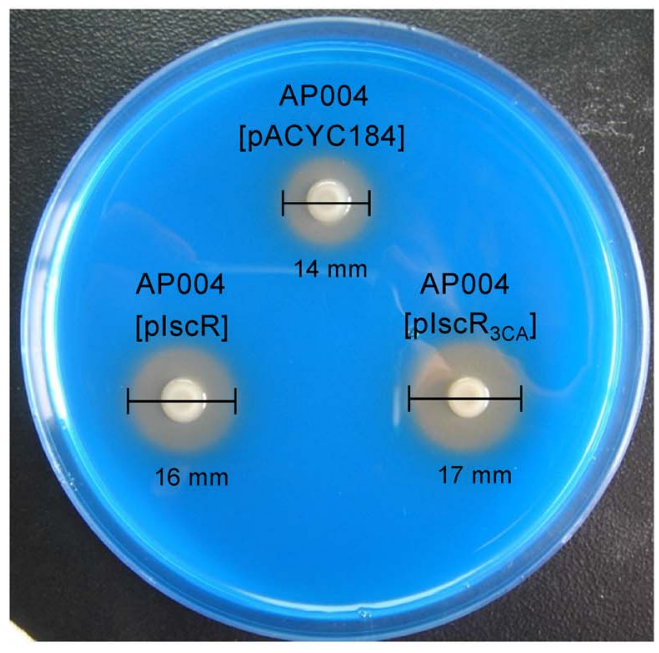

Figure 4. Deletion of iscR decreases $K$. pneumoniae $\Delta$ fur siderophore production assessed using CAS assay. All assayed bacterial mutants (A) and the complement strains (B) are indicated. The halos around the colonies correspond to the iron-chelating activity of siderophores in bacteria were measured after $24 \mathrm{~h}$ of incubation at $37^{\circ} \mathrm{C}$. The assay was independently repeated at least five times, and the differences among strains are consistent.

doi:10.1371/journal.pone.0107812.g004

using a LacZ reporter system [23]. As shown in Fig. 6A, the addition of 250 or $500 \mu \mathrm{M}$ Dip to the growth medium increased iscR promoter $\left(\mathrm{P}_{i s c R}\right)$ activity by approximately 2 -fold and 3.9 -fold, respectively, indicating that the transcription of the iscRSUA operon was activated by iron limitation.

In K. pneumoniae, Fur and RyhB reportedly play crucial roles in gene regulation in response to cellular iron levels $[10,11]$. Thus, we investigated whether Fur and RyhB regulate the activity of $\mathrm{P}_{\text {iscR. }}$. As shown in Fig. $6 \mathrm{~B}$, the deletion of fur and the further deletion of $r y h B$ in AP006 strain had no obvious effects on $\mathrm{P}_{\text {iscR }}$ activity, whereas the deletion of iscR in AP006 strain activated $\mathrm{P}_{\text {isc } R}$ activity by approximately 4.5 -fold. In E. coli, IscR has been demonstrated to exert negative auto-regulation which requires the [2Fe-2S] cluster [20]. Thus, we measured $\mathrm{P}_{i s c R}$ activity in an AP006-derived strain, AP008 ( $\Delta l a c Z$-isc $\left.R_{3 \mathrm{CA}}\right)$, expressing a mutated IscR predicted to be defective in cluster binding [20]. As shown in Fig. $6 \mathrm{~B}$, the $\mathrm{P}_{\text {isc } R}$ activity in AP008 was increased approximately 4.5 -fold. This increase was comparable to that in AP007 $(\Delta l a c Z-\Delta i s c R)$. Our results suggested that IscR inhibits the

Table 1. qRT-PCR analyses of the expression of iron-acquisition genes in $K$. pneumoniae strains.

\begin{tabular}{|c|c|c|c|c|}
\hline \multirow[t]{2}{*}{ Systems } & \multirow[t]{2}{*}{ Gene } & \multicolumn{3}{|c|}{ RNA expression ratio ${ }^{b}$} \\
\hline & & AP005/AP004 ${ }^{a}$ & 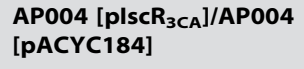 & AP004 [plscR]/AP004 [pACYC184] \\
\hline \multicolumn{5}{|l|}{$\mathrm{Fe}^{3+}$} \\
\hline Ferrichrome & fhuA & $2.17 \pm 0.31$ & $2.83 \pm 0.14$ & $1.78 \pm 0.42$ \\
\hline Aerobactin & iucA & $2.24 \pm 0.12$ & $3.23 \pm 0.15$ & $1.84 \pm 0.22$ \\
\hline \multirow[t]{3}{*}{ Enterobactin } & fepA & $1.07 \pm 0.38$ & $N D^{c}$ & ND \\
\hline & fepB & $1.11 \pm 0.09$ & ND & ND \\
\hline & entC & $0.80 \pm 0.09$ & ND & ND \\
\hline \multirow[t]{2}{*}{ Ferric citrate } & fec $A$ & $1.23 \pm 0.52$ & ND & ND \\
\hline & fecE & $1.74 \pm 0.25$ & ND & ND \\
\hline Salmochelin & $\operatorname{iroB}$ & $1.92 \pm 0.13$ & ND & ND \\
\hline Heme & hmuR & $1.58 \pm 0.22$ & ND & ND \\
\hline \multicolumn{5}{|l|}{$\mathrm{Fe}^{2+}$} \\
\hline \multirow[t]{2}{*}{ Ferrous iron } & $f e o B$ & $1.35 \pm 0.36$ & ND & ND \\
\hline & sitA & $3.16 \pm 0.01$ & $3.68 \pm 0.11$ & $2.27 \pm 0.23$ \\
\hline
\end{tabular}

${ }^{a}$ AP004, CG43S3 fur- $\Delta i s c R ; A P 005, C G 43 S 3 \Delta$ fur-iscR 3 CA.

${ }^{b}$ Mean expression ratio ( \pm SD) of AP005 relative to AP004, AP004 [plscR 3 CA] relative to AP004 [pACYC184], or AP004 [plscR] relative to AP004 [pACYC184]

c ND, not determined.

doi:10.1371/journal.pone.0107812.t001 


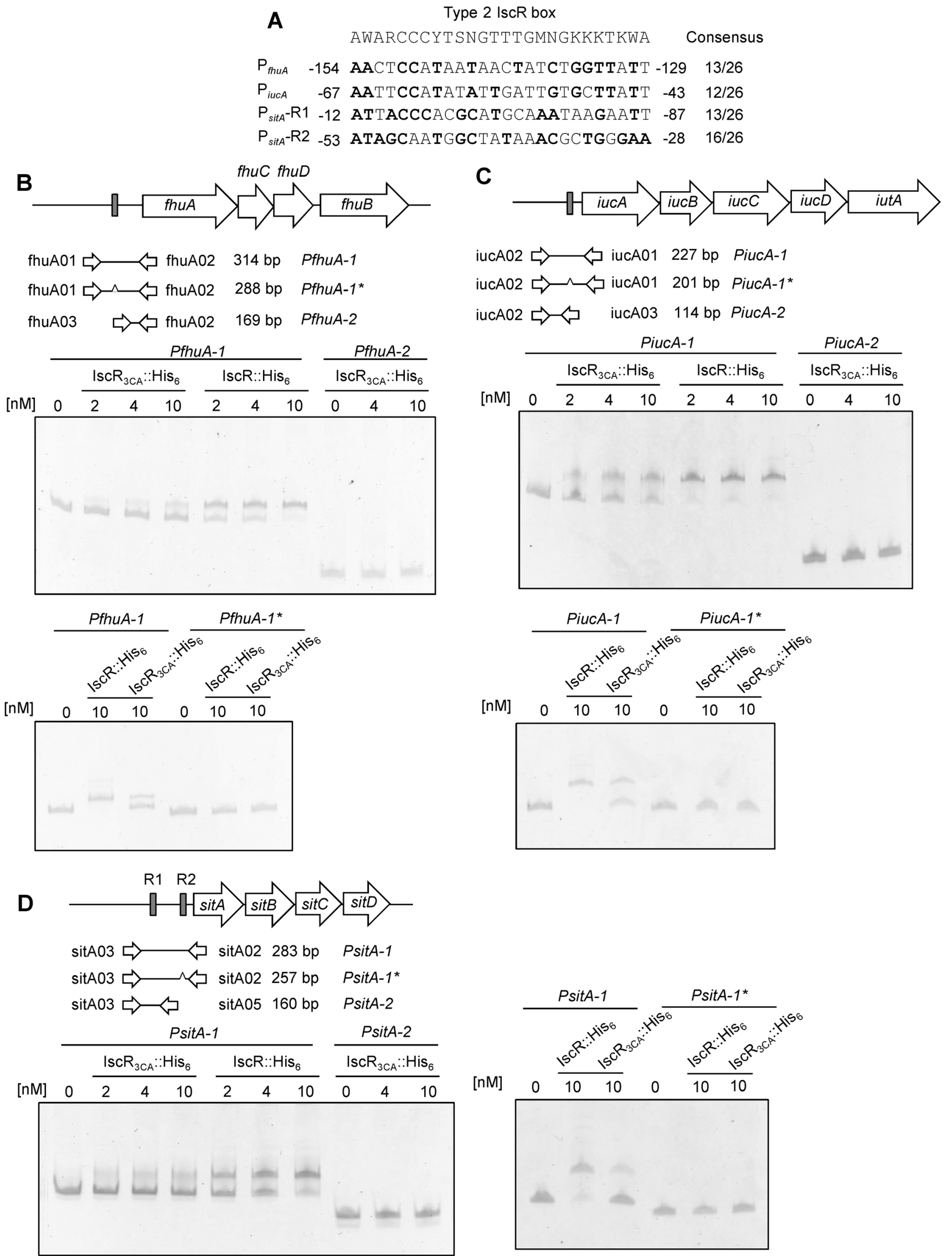


Figure 5. IscR $\mathbf{R}_{\mathbf{3 C A}}:$ : His $\mathbf{6}$ binds directly to $\mathbf{P}_{\text {fhuA }}, \mathbf{P}_{\text {iucA }}$, and $\mathbf{P}_{\text {sita. }}$. (A) DNA sequence alignment between the $E$. coli type 2 IscR box and the putative IsCR binding sequence in the upstream regions of fhuA, iucA, and sitA. Positions identical to the consensus sequences are bolded. Diagrammatic representation of the fhuA (B), iucA (C), and sitA (D) loci. The large arrows represent the open reading frames. The primer sets used in PCR amplification of the DNA probes are indicated, and the numbers denote the DNA amplified length. The predicted IscR boxes is deleted and indicated

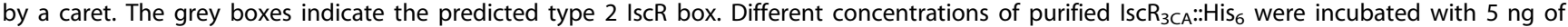
various DNA fragments of the upstream regions of indicated genes. Following incubation at room temperature for 30 min, the mixtures were analysed on a $5 \%$ non-denaturing polyacrylamide gel. The gel was stained with SYBR Green I dye and photographed.

doi:10.1371/journal.pone.0107812.g005

transcription of the iscRSUA operon in a [2Fe-2S] clusterdependent manner in K. pneumoniae.

To investigate whether IscR is the sole regulator of iscRSUA transcription in response to iron availability, we monitored $\mathrm{P}_{i s c R}$ activity in the AP007 and AP008 strains in LB broth containing various levels of iron. As shown in Fig. $6 \mathrm{C}$, the $\mathrm{P}_{\text {isc }}$ activity in AP006 was activated in LB broth supplemented with Dip, and the further addition of 100 or $250 \mu \mathrm{M} \mathrm{FeSO}_{4}$ reversed the activation. In the AP007 and AP008 strains, $\mathrm{P}_{\text {isc }}$ activity was increased compared with that of AP006. Nevertheless, the addition of Dip still activated $\mathrm{P}_{i s c R}$ activity, suggesting the presence of unknown factors, and the further addition of $\mathrm{FeSO}_{4}$ restored the effect.

To analyse whether Fur is responsible for this regulation, we measured $\mathrm{P}_{i s c R}$ activity in AP011 ( $\left.\Delta l a c Z-\Delta f u r-\Delta i s c R\right)$ compared with that of AP007 at various iron levels and noted no obvious effect. These results indicated that in addition to IscR and Fur, other factors modulate the transcription of the iscRSUA operon in response to environmental iron availability.

\section{Discussion}

Clinically isolated $K$. pneumoniae strains usually carry large amounts of CPS to resist engulfment by phagocytes and serum bactericidal factors [6,24]. Therefore, tightly controlling CPS biosynthesis is critical for successful infection by $K$. pneumoniae $[10,11,25]$. We have previously shown that Fur represses the expression of mucoid factors RmpA and RcsA as well as the small RNA RyhB in response to environmental iron to decrease CPS biosynthesis indirectly in K. pneumoniae [10,11]. In this study, we focused on IscR, a central regulator of iron metabolism, to analyse more thoroughly how external iron affects CPS biosynthesis. Our data indicated that IscR activates CPS biosynthesis in a Fe-S cluster-dependent manner (Fig. 1A and B). Moreover, IscR positively regulates the transcription of three transcription units in the $c p s$ gene cluster (Fig. 1G). Purified IscR::His 6 also directly interacts with the promoter of orf1-2, possibly through the predicted type 1 IscR box (Fig. 2). These findings indicated that Fur and IscR exert negative and positive regulation, respectively, on CPS biosynthesis in response to external iron.

However, we hypothesize herein that Fur plays a major regulatory role because (i) $K$. pneumoniae grown under ironreplete conditions displayed decreased CPS levels, (ii) the AP004 strain produced elevated amounts of CPS compared with that of the WT and in the AP004 background, expression of IscR or $\mathrm{Isc}_{3 \mathrm{CA}}$ did not cause an obvious effect on CPS amount under iron-replete or iron-limited conditions (data not shown), and (iii) all three transcription units of the $c p s$ genes were obviously increased after fur deletion [10]. This result supported the notion that Fur plays a major regulatory role in the regulation of CPS biosynthesis. Nevertheless, the contribution of IscR could also be an important part of the iron network. On one hand, in iron-replete conditions, Fur indirectly represses the CPS production, however CPS is still needed for $K$. pneumoniae survival inside the host, so IscR may contribute in maintaining a higher basal expression level of the genes involved in CPS biosynthesis. On the other hand, in ironlimited conditions, the transcription of iron-acquisition genes is increased not only due to the de-repression of apo-Fur but also the activation of apo-IscR. Besides, in addition to iron level, oxidative stress has been demonstrated to signal Fur and IscR, and the reversible interconversion of $\mathrm{Fe}-\mathrm{S}$ clusters makes them exquisite sensors of such stress $[13,18,26]$. Thus, the modulation of CPS levels by Fur and IscR under oxidative stress conditions could be predicted, but this hypothesis remains to be elucidated.

A BLAST search identified eight putative iron acquisition systems in the genome of $K$. pneumoniae CG43S3. Moreover, Fur directly represses the transcription of genes corresponding to six iron acquisition systems (iucA, iroB, entC, hmuR, feoA, and fecA) under iron-replete conditions [10]. In this study, we have shown that IscR directly activates the transcription of $f h u A$, sitA, and iucA (Table 1 and Fig. 5). Although fur deletion also led to increased expression of these three iron acquisition systems, an in vivo Fur titration assay demonstrated that Fur did not interact with the promoters of $f h u A$ and sitA and also had a relatively lower binding affinity to iucA [10]. These findings indicated that Fur and IscR orchestrate the expression of iron acquisition systems in $K$. pneumoniae. In addition, Fur is suggested to play a major regulatory role because deletion of fur, but not iscR deletion, activated the secretion of siderophores, and the effect mediated by IscR was observed only in the fur-deleted background (Fig. 4).

Because IscR and Fur are both iron-responsive regulators which share strong overlap among downstream targets, we next investigated whether IscR and Fur are cross-regulated. In E. coli, the expression of the iscR is controlled not by Fur but by IscR itself $[19,20]$. In $K$. pneumoniae, we found that $\mathrm{P}_{\text {isc } R}$ activity is clearly activated by IscR but not influenced by Fur (Fig. 6), which is consistent to the finding in E. coli. Moreover, deletion of $i s c R$ did not affect the transcription of fur (data not shown), suggesting that in K. pneumoniae, IscR and Fur do not share cross-regulation. Furthermore, E. coli RyhB reportedly binds directly to the upstream region of isc $S$ mRNA to decrease the stability of iscRSUA but not iscR mRNA, thereby leading to a stable secondary isc $R$ structure and resulting in active translation [27]. Then, the increased IscR likely causes auto-repression of $\mathrm{P}_{i s c R}$ activity.

An analysis of the upstream region of isc $S$ in K. pneumoniae, also identified a conserved sequence paired with RyhB (data not shown). However, the regulatory effect on $\mathrm{P}_{i s c R}$ activity mediated by RyhB was not obvious under our assay conditions (Fig. 6B). On the contrary, as shown in Fig. $6 \mathrm{C}, \mathrm{P}_{i s c R}$ activity in AP007 and AP008 was still activated by iron depletion, which prompted us to verify whether IscR is the sole iron-responsive regulator that controls $\mathrm{P}_{i s c R}$ activity. However, in $\mathrm{AP007}$, the addition of $\mathrm{FeSO}_{4}$ to iron-depleted medium still led to a reduction in $\mathrm{P}_{i s c R}$ activity. These results suggested that an unknown regulator, beside of IscR and Fur, represses the transcription of isc $R$ in response to external iron. In addition to IscR, the [2Fe-2S] cluster is critical for regulation mediated by FNR and SoxR [27]. However, sequence analysis of the promoter region of $i s c R$ revealed no typical FNR and SoxR binding sites. On the contrary, we found a putative binding site of SoxS [28], an oxidative transcriptional regulator activated by SoxR, in $\mathrm{P}_{\text {isc }}$. This putative SoxS binding site displays $79 \%$ (15/19 bp) homology with the consensus sequence 
A

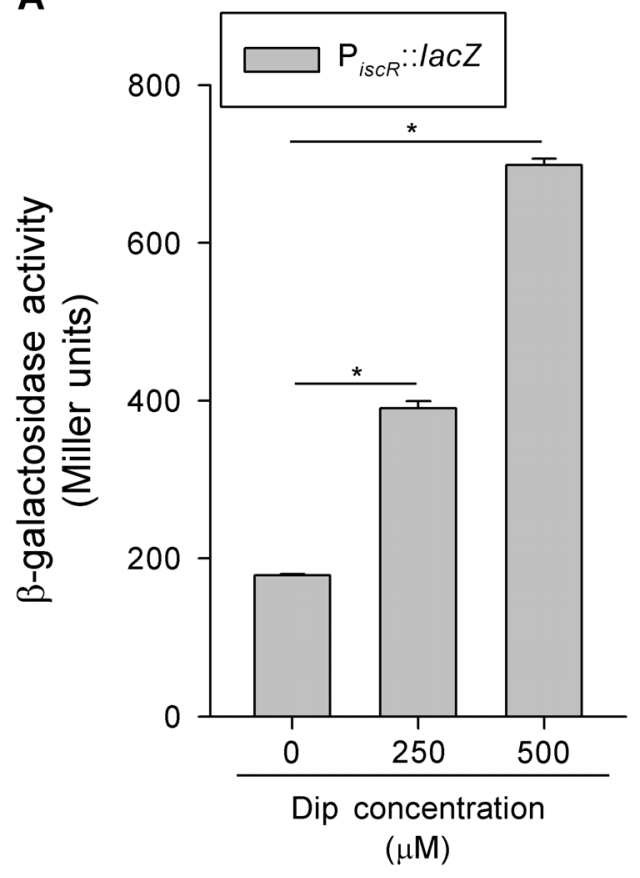

B

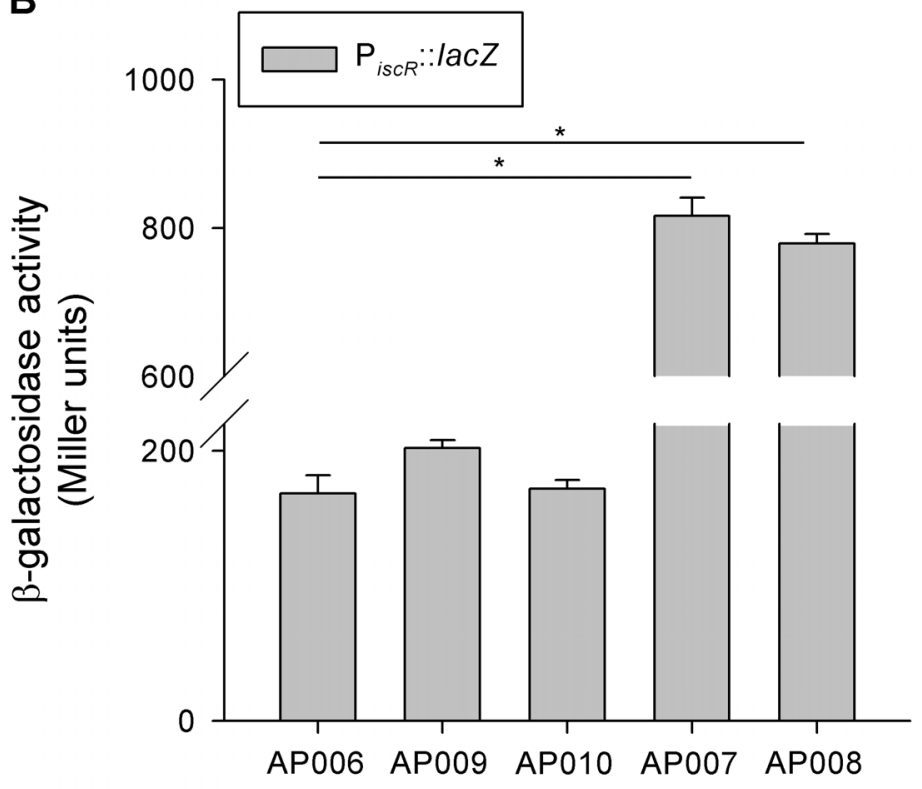

C LB

$\mathrm{LB}+250 \mu \mathrm{M}$ Dip

$\mathrm{LB}+250 \mu \mathrm{M}$ Dip+100 $\mu \mathrm{M}$ FeSO4

$\mathrm{LB}+250 \mu \mathrm{M}$ Dip+250 $\mu \mathrm{M}$ FeSO4



Figure 6. Regulation of $K$. pneumoniae iscR expression. (A) $\beta$-Galactosidase activity of $K$. pneumoniae AP006 ( $\Delta$ lacZ) carrying the reporter

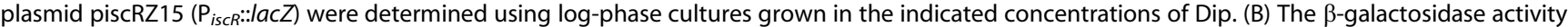


is $\left.C R_{3 C A}\right)$, using log-phase cultures grown in LB medium. (C) The $\beta$-galactosidase activity of piscRZ15 was determined in the AP006 and isogenic strains, AP007, AP008, and AP011 ( $\Delta / a c Z-\Delta$ fur- $\Delta i s c R$ ), using log-phase cultures grown in the indicated media. Error bars indicate standard deviations. ${ }^{*} P<0.01$ compared with the indicated group.

doi:10.1371/journal.pone.0107812.g006 
and is located at position -144 to -126 relative to the translation start site of $i s c R$. Therefore, we hypothesized that SoxS may be involved in the regulation of $i s c R$ in response to oxidative stress and will investigate this possibility in future studies.

IscR differs from other known Fe-S cluster-containing transcription factors such as FNR and SoxR because both apo- and holo-IscR regulate transcription and exhibit different DNA binding specificities [29]. Structural and biochemical studies have suggested that the ligation of the $[2 \mathrm{Fe}-2 \mathrm{~S}]$ cluster broadens the DNA binding specificity of IscR, thereby allowing holo-IscR to bind both type 1 and type 2 boxes, whereas apo-IscR binds only the type 2 box [29]. In the K. pneumoniae K2 cps gene cluster, we found a type 1 box in the promoter region of galF, and purified IscR::His ${ }_{6}$ could bind this motif in vitro (Fig. 2). In addition and as expected, the clusterless $\mathrm{IscR}_{3 \mathrm{CA}}: \mathrm{His}_{6}$, mimicking apo-IscR, showed no obvious binding affinity to $\mathrm{P}_{\text {galF }}$. On the contrary, all three promoters of the iron acquisition genes regulated by IscR contain predicted type 2 boxes, and both IscR::His ${ }_{6}$ and $\mathrm{IscR}_{3 \mathrm{CA}}: \mathrm{His}_{6}$ appeared to bind these boxes (Fig. 5). Although apo- and holo-IscR have been demonstrated to bind the type 2 box with similarly high affinity [21], EMSA revealed that $\mathrm{IscR}_{3 \mathrm{CA}}: \mathrm{His}_{6}$ displayed weaker binding (Fig. 5). In E. coli, the $\mathrm{Fe}-\mathrm{S}$ cluster status of IscR is a key variable that regulates gene expression in response to iron availability [12]. Our results suggested that the transcription of $c p s$ and iron acquisition genes is regulated by not only the level of IscR but also the cellular ratio of apo- and holo-IscR.

Although IscR was first discovered as an auto-repressor of the isc operon, it is now known to be a global regulator that influences the expression of $\sim 40$ genes in E. coli and $\sim 67$ genes in Vibrio vulnificus $[19,30]$. Although the regulon of IscR in K. pneumoniae has not been identified, our study provided evidence that IscR regulates CPS biosynthesis and iron acquisition, which are also regulated by Fur. Moreover, previous studies have shown that Fur controls type 3 fimbriae expression and biofilm formation in $K$. pneumoniae [31]. The possibility that Isc R also participates in the regulation of these phenotypes is currently under investigation.

After infection, bacterial pathogens frequently encounter iron starvation and oxidative/nitric oxide stress conditions that are highly detrimental for maintaining $\mathrm{Fe}-\mathrm{S}$ cluster homeostasis $[32,33,34]$. These conditions are predicted to influence the transcription of genes regulated by IscR. Thus, in response to these stress conditions, IscR may regulate CPS biosynthesis, iron acquisition systems, and other virulence factors in $K$. pneumoniae to facilitate bacterial persistence in the host. In this study, we demonstrated that external iron levels regulate CPS biosynthesis and iron acquisition systems through IscR in K. pneumoniae and proposed a working model (Fig. 7). In response to iron availability, IscR and Fur control the expression of downstream targets in a parallel and cooperative manner, which is predicted to play a crucial regulatory role during infection.

\section{Materials and Methods}

\section{Bacterial strains, plasmids, and media}

Bacterial strains and plasmids used in this study are listed in Table 2. Primers used in this study are list in Table 3. Bacteria were routinely cultured at $37^{\circ} \mathrm{C}$ in $\mathrm{LB}$ medium supplemented with appropriate antibiotics. The antibiotics used include ampicillin $(100 \mu \mathrm{g} / \mathrm{ml})$, kanamycin $(25 \mu \mathrm{g} / \mathrm{ml})$, streptomycin $(500 \mu \mathrm{g} / \mathrm{ml})$, and tetracycline $(12.5 \mu \mathrm{g} / \mathrm{ml})$.

\section{Construction of the deletion of iscR mutants}

Specific gene deletion of iscR was introduced into $K$. pneumoniae CG43S3 using an allelic exchange strategy as previously described [35]. In brief, two approximately $1000 \mathrm{bp}$ DNA fragments flanking both sides of $i s c R$ were cloned into the suicide vector pKAS46 [36], a suicide vector containing rpsL, which allows positive selection with streptomycin for vector loss. The resulting plasmid was then mobilized from E. coli $\mathrm{S} 17-1 \lambda$ pir [37] to K. pneumoniae CG43S3 or CG43S3-derived strains by conjugation. The transconjugants, with the plasmid integrated into the chromosome via homologous recombination, were selected with ampicillin and kanamycin on M9 agar plates. Several of the colonies were grown in LB broth supplemented with $500 \mu \mathrm{g} / \mathrm{mL}$ of streptomycin to log phase at $37^{\circ} \mathrm{C}$ and then spread onto an $\mathrm{LB}$ agar plate containing $500 \mu \mathrm{g} / \mathrm{mL}$ of streptomycin. The streptomycin-resistant and kanamycin-sensitive colonies were selected, and the deletion was verified by PCR and Southern hybridization (data not shown). The resulting $K$. pneumoniae mutants are listed in Table 2.

\section{Construction of the plscR complementation plasmid and the plscR $R_{3 C A}$ mutant plasmid}

To obtain the complementation plasmid (pIscR), a DNA fragment containing the promoter and coding sequence of $i s c R$ was amplified by PCR using the primer pair GT138/GT139 (Table 3) and cloned into the pACYC184 shuttle vector. The pIsc $\mathrm{R}_{3 \mathrm{CA}}$ plasmid, which carried the mutant allele encoding IscR with the C92A, C98A, and C104A mutations, was constructed using the inverse-PCR method. Briefly, the pIscR plasmid was used as the PGR template to generate the mutant allele with the primer pair GT206/GT207 (Table 3). The recovered PCR product was treated with $D p n \mathrm{I}$ for $2 \mathrm{~h}$, subjected to $\mathrm{T} 4$ polynucleotide kinase treatment, and self-ligated with T4 DNA ligase. The ligation product was transformed into E. coli $\mathrm{DH} 5 \alpha$. The $\mathrm{IscR}_{3 \mathrm{CA}}$ plasmid was subsequently confirmed by sequence analysis.

\section{Construction of a $K$. pneumoniae isc $R_{3 C A}$ mutant}

A DNA fragment carrying iscR and approximately $1000 \mathrm{bp}$ adjacent regions on either side was amplified by PCR using primer pairs GT241/GT242 (Table 3) and cloned into yT\&A. The resulting plasmid was used as the template for the inverse-PCR with the primer pair GT206/GT207 (Table 3) to generate a mutant iscR allele encoding the C92A, C98A and C104A mutations. Subsequently, the mutant allele of isc $R$ was subcloned into pKAS46 and confirmed by DNA sequencing. Then, the plasmid was mobilized from E. coli S17-1 $\lambda$ pir to the $K$. pneumoniae AP001 strain by conjugation, and the subsequent selection was performed as described above.

\section{Extraction and quantification of CPS}

CPS was extracted and quantified as previously described [38]. The glucuronic acid content, represents the amount of $K$. pneumoniae K2 CPS, was determined from a standard curve of glucuronic acid (Sigma-Aldrich) and expressed as micrograms per $10^{9}$ c.f.u. [39].

\section{Measurement of promoter activity}

The promoter-reporter plasmids, pOrf12, pOrf315, pOrf1617, and piscRZ15 were individually mobilized into K. pneumoniae strains by conjugation from E. coli S17-1 $\lambda$ pir. The bacteria were grown to logarithmic phase in LB broth or indicated medium, and 



Type I IscR box \Type 2 IscR box

Figure 7. A proposed model for IscR regulation on the CPS biosynthesis and iron-acquisition genes in $K$. pneumoniae. (A) Under ironreplete conditions, and when the cellular supply of Fe-S clusters is sufficient, the holo-IscR exerts an auto-repression as well as activates the K2 cps genes and the iron-acquisition genes; however, Fur in complex with Fe(II) exerts a stronger repression on these genes (red lines). (B) Under irondeplete conditions, the apo-IscR accumulates and preferably activates the transcription of the iron-acquisition genes but not the $c p s$ genes. Upon the de-repression of Fur, both the transcription of the iron-acquisition and cps genes are increased. doi:10.1371/journal.pone.0107812.g007

the $\beta$-galactosidase activity was measured as previously described [23].

\section{Bacterial survival in serum}

Normal human serum, pooled from healthy volunteers, was divided into equal volumes and stored at $-70^{\circ} \mathrm{C}$ before use. Bacterial survival in serum was determined as previously described [35]. First, the bacteria were grown to log phase in LB broth and the viable bacterial concentration was adjusted to $1 \times 10^{6}$ c.f.u. $/ \mathrm{ml}$. Next, $1 \mathrm{ml}$ of the cultures was washed twice using phosphatebuffered saline (PBS) and resuspended in $1 \mathrm{ml}$ PBS. A mixture containing $250 \mu \mathrm{l}$ of the cell suspension and $750 \mu \mathrm{l}$ of pooled human serum was incubated at $37^{\circ} \mathrm{C}$ for $15 \mathrm{~min}$. The number of viable bacteria was then determined by plate counting. The survival rate was expressed as the number of viable bacteria treated with human serum compared with the number of viable bacteria pretreatment. The $0 \%$ survival of $K$. pneumoniae AP012 $(\Delta$ galU $)$ served as a negative control.

\section{Purification of IscR::His ${ }_{6}$ and IscR $\mathrm{R}_{3 \mathrm{CA}}:: \mathrm{His}_{6}$}

The coding regions of $i s c R$ and $i s c R_{3 \mathrm{CA}}$ were amplified using the primer pair GT215/GT216 (Table 3) and cloned into the NheI/XhoI site in pET30b (Novagen, 205 Madison, Wis). The resulting plasmids (pET30b-IscR and pET30b-IscR $\mathrm{R}_{3 \mathrm{CA}}$, respectively) were then transformed into $E$. coli BL21(DE3)[pLysS] (Invitrogen, USA), and overproduction of the recombinant proteins IscR::His ${ }_{6}$ and $\operatorname{IscR}_{3 \mathrm{CA}}:: \mathrm{His}_{6}$, respectively, were induced by the addition of $1 \mathrm{mM}$ IPTG for $3 \mathrm{~h}$ at $37^{\circ} \mathrm{C}$. The cell pellets were washed and resuspended in cold binding buffer $(20 \mathrm{mM}$ sodium phosphate, $0.5 \mathrm{M} \mathrm{NaCl}, 5 \mathrm{mM}$ imidazole, $\mathrm{pH}$ 7.4). The cells were then broken by sonication and the cell pellets were removed by centrifugation at $14000 \mathrm{rpm}$ for $10 \mathrm{~min}$ at $4^{\circ} \mathrm{C}$. The recombinant proteins were then purified from the soluble fraction of the total cell lysate by affinity chromatography using His-Bind resin (Novagen, Madison, Wis) according to the manufacturer's instructions. The nonbinding proteins were washed away using binding buffer and the recombinant proteins were eluted by elution buffer $(20 \mathrm{mM}$ sodium phosphate, $0.5 \mathrm{M} \mathrm{NaCl}, 500 \mathrm{mM}$ imidazole, $\mathrm{pH}$ 7.4). Finally, the purified proteins were dialyzed against TEGX buffer [20 mM Tris-HCl ( $\mathrm{pH}$ 8.0), $0.5 \mathrm{mM}$ EDTA $(\mathrm{pH} 8.0), 10 \%$ (v/v) glycerol, $0.2 \%$ Triton-X 100] containing $0.1 \mathrm{mM} \mathrm{NaCl}$ at $4{ }^{\circ} \mathrm{C}$ overnight. Subsequently, the dialyzed protein was checked for purity by SDS-PAGE and stored for up to two weeks at $4^{\circ} \mathrm{C}$. The purified protein was transparent and no obvious precipitation was observed after storage.

\section{EMSA}

DNA fragments of the putative promoter regions galF, fhuA, $i u c A$, and sitA were amplified with Pfu polymerase using specific primer sets (Table 3 ) to generate DNA probes for EMSA (PgalF-1 and PgalF-2 for galF; PfhuA-1 and PfhuA-2 for fhuA; PiucA-1 and PiucA-2 for iucA; and PsitA-1 and PsitA-2 for sitA). To obtain probes that lacked a putative IscR box, the DNA fragments were respectively amplified with Taq polymerase using the abovedescribed primer sets (Table 3 ) and then cloned into yT\&A to generate the plasmids pgalF, pfhuA, piucA, and psitA for subsequent mutagenesis via the inverse-PCR method using the primer pairs GT201/galF01, fhuA04/fhuA05, iucA03/iucA04, 
Table 2. Bacterial strains and plasmids used in this study.

\begin{tabular}{|c|c|c|}
\hline Strains or plasmids & Descriptions & Reference or source \\
\hline \multicolumn{3}{|l|}{ K. pneumoniae } \\
\hline CG43S3 & CG43 Sm ${ }^{r}$ & [41] \\
\hline AP001 & CG43S3 3 iscR & This study \\
\hline AP002 & CG43S3isc $R_{3 C A}$ & This study \\
\hline AP003 & CG43S3 $\Delta$ fur & [42] \\
\hline AP004 & CG43S3 $\Delta$ fur- $\Delta i s c R$ & This study \\
\hline AP005 & CG43S3 $\Delta$ fur-isc $R_{3 C A}$ & This study \\
\hline AP006 & CG43S3 $\Delta / a c Z$ & [23] \\
\hline AP007 & CG43S3 $\Delta / a c Z-\Delta i s c R$ & This study \\
\hline AP008 & CG43S3 3 lacZ-isc $R_{3 C A}$ & This study \\
\hline AP009 & CG43S3Alacz- $\Delta$ fur & This study \\
\hline AP010 & CG43S3 $\Delta$ lacZ- $\Delta$ fur- $\Delta r y h B$ & This study \\
\hline AP011 & CG43S3 $\Delta$ lacZ- $\Delta$ fur- $\Delta i s c R$ & This study \\
\hline AP012 & CG43S3 3 galu & [35] \\
\hline \multicolumn{3}{|l|}{ E. coli } \\
\hline $\mathrm{DH} 5 \alpha$ & 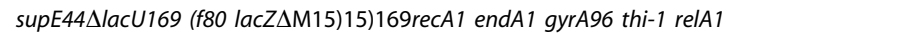 & [43] \\
\hline BL21(DE3) & F ompT hsdS $\left.{ }_{B} r_{B}^{-} m_{B}^{-}\right] g a l d c m[D E 3]$ & New England Biolabs \\
\hline $\mathrm{S} 17-1 \lambda$ pir & hsdR recA pro RP4-2 [Tc::Mu; Km:::Tn7] [ipir] & [37] \\
\hline \multicolumn{3}{|l|}{ Plasmids } \\
\hline pKAS46 & $\mathrm{Ap}^{r} \mathrm{Km}^{r}$, positive selection suicide vector, $r p s L$ & [36] \\
\hline yT\&A & $A p^{r}$, TA cloning vector & Yeastern \\
\hline pACYC184 & $T c^{r} \mathrm{Cm}^{r}$, low copy number cloning vector & New England Biolabs \\
\hline plscR & $\mathrm{Cm}^{r}, 980$-bp fragment containing an isc $R$ allele cloned into pACYC184 & This study \\
\hline plscR $R_{3 C A}$ & $\begin{array}{l}\mathrm{Cm}^{\mathrm{r}} \text {, 980-bp fragment containing C92A, C98A and C104A mutant allele } \\
\text { of iscR cloned into pACYC184 }\end{array}$ & This study \\
\hline placZ15 & $\mathrm{Cm}^{r}$, promoter selection vector, lac $\mathrm{Z}^{+}$ & [23] \\
\hline piscRZ15 & $\mathrm{Cm}^{\mathrm{r}}, 312$-bp fragment containing the region upstream of isc $R$ cloned into placZ15 & This study \\
\hline pOrf12 & $\begin{array}{l}\mathrm{Cm}^{\mathrm{r}} \text {, 500-bp fragment containing the region upstream of Klebsiella K2 cps orf1-orf2 } \\
\text { cloned into placZ15 }\end{array}$ & [23] \\
\hline pOrf315 & $\begin{array}{l}\mathrm{Cm}^{r} \text {, 900-bp fragment containing the region upstream of Klebsiella K2 cps orf3-orf15 } \\
\text { cloned into placZ15 }\end{array}$ & [23] \\
\hline pOrf1617 & $\begin{array}{l}\mathrm{Cm}^{\mathrm{r}}, 300 \text {-bp fragment containing the region upstream of Klebsiella K2 cps } \\
\text { orf16-orf17 cloned into placZ15 }\end{array}$ & [23] \\
\hline pET30b-IscR & $\mathrm{Km}^{\mathrm{r}}$, 654-bp fragment encoding full-length IscR cloned into pET30b & This study \\
\hline pET30b-IscR 3 CA & $\begin{array}{l}\mathrm{Km}^{\mathrm{r}} \text {, 654-bp fragment encoding full-length C92A, C98A and C104A mutant allele } \\
\text { of iscR cloned into pET30b }\end{array}$ & This study \\
\hline piscR-pKAS46 & $\mathrm{Ap}^{\mathrm{r}} \mathrm{Km}^{\mathrm{r}}, 2.0 \mathrm{~kb}$ fragment containing isc $R$ and its flanking regions cloned into pKAS46 & This study \\
\hline piscR 3 CA-pKAS46 & $\begin{array}{l}\mathrm{Ap}^{r} \mathrm{Km}^{\mathrm{r}}, 2.0 \mathrm{~kb} \text { fragment containing full-length C92A, C98A and C104A mutant allele } \\
\text { of is } \mathrm{C} R \text { and its flanking regions cloned into pKAS46 }\end{array}$ & This study \\
\hline
\end{tabular}

doi:10.1371/journal.pone.0107812.t002

and sitA05/sitA06, respectively. The resulting mutant plasmids, pgalF*, pfhuA*, piucA*, and psitA*, respectively, were amplified with specific primer sets to generate the DNA fragments PgalF$1^{*}, P$ PhuA $-1^{*}, \operatorname{PiucA}-1^{*}$, and PsitA-1* for DNA probes in EMSA.

For the EMSA, the purified IscR::His $\mathrm{His}_{6}$ and $\mathrm{IscR}_{3 \mathrm{CA}}:: \mathrm{His}_{6}$ proteins were incubated with 5-ng DNA in a $10 \mu \mathrm{l}$ solution containing $4 \mathrm{mM}$ Tris-HCl (pH 7.4), $10 \mathrm{mM} \mathrm{KCl,} 100 \mathrm{mM}$ dithiothreitol, and $10 \mu \mathrm{g} / \mathrm{ml} \mathrm{BSA}$ at $37^{\circ} \mathrm{C}$ for $30 \mathrm{~min}$. The samples were then loaded onto a native gel of $5 \%$ nondenaturing polyacrylamide in $0.5 \times \mathrm{TB}$ buffer $(45 \mathrm{mM}$ Tris-HCl, $\mathrm{pH} 8.0$, $45 \mathrm{mM}$ boric acid). Gels were electrophoresed with a $20-\mathrm{mA}$ current at $4^{\circ} \mathrm{C}$ and then stained with SYBR Green I dye (Invitrogen). The assay was repeated in at least 3 independent experiments.

\section{qRT-PCR}

Total RNAs were isolated from early-exponential-phase grown bacteria cells by use of the RNeasy midi-column (QIAGEN) according to the manufacturer's instructions. RNA was DNasetreated with RNase-free DNase I (MoBioPlus) to eliminate DNA contamination. RNA of 100-ng was reverse-transcribed with the Transcriptor First Strand cDNA Synthesis Kit (Roche) using random primers. qRT-PCR was performed in a Roche 
Table 3. Primers used in this study.

\begin{tabular}{|c|c|c|c|}
\hline Primer & Sequence $\left(5^{\prime} \rightarrow 3^{\prime}\right)$ & Enzyme cleaved & \\
\hline GT138 & GGATCCTGCGTCGTCTGTTCACCC & BamHI & \\
\hline GT139 & AAGCTTCAATGCAAGGAATCAGGCA & HindllI & \\
\hline GT142 & GGATCCCGGTCACGGCATAA & BamHI & \\
\hline GT143 & AGATCTAGTTGAACATCCTGCGCGGC & Bg/ll & \\
\hline GT206 & CTGAGCGCCGCCCTTGCCCTGGGCACG & & \\
\hline GT207 & GGCGGCGATAAAGCCCTGACTCACG & & \\
\hline GT215 & GCTCGAGGGCGCGCAGTTTAACGTCAAT & Nhel & \\
\hline GT216 & GCATATGAGACTGACATCTAAAGGGCG & Xhol & \\
\hline GT241 & CGAGCTCCCATACCGGCAACATGGG & Sacl & \\
\hline GT242 & СTCTAGACGCGCCATCTCGCTITCC & Xbal & \\
\hline $\mathrm{CCO} 2$ & GGATCCTCTCATGTCTTACTTAACC & BamHI & \\
\hline $\mathrm{CCO}$ & GGATCCAGGACGCGATTGAC & BamHI & \\
\hline GT200 & TCAGCGGCTCGTTCCTTTGC & & \\
\hline GT201 & TCTCACTTCTGACTGTTGGTAAAAGGG & & \\
\hline YCCO2 & ACTGGATCCTGCGACCGGAATAACC & BamHI & \\
\hline fhuA01 & GAAGCTTGTCGCGGGCTGGATCAAG & HindllI & \\
\hline fhuA02 & CGGATCCCGCAGCGAGTGATTTGGC & BamHI & \\
\hline fhuA03 & AATTCAGCCTTCGACCGGC & & \\
\hline fhuA04 & AAAGAGCCGTTAACTTTTTTAC & & \\
\hline fhuA05 & TAATTCAGCCTTCGACCGG & & \\
\hline iucA01 & GGCAAAGTCATAGTCAAACAGATC & & \\
\hline iucA02 & GAATCACCAGTACAGGGATCG & & \\
\hline iucA03 & ACAACATCGTGACGTCTCTTGA & & \\
\hline iucA04 & ТTATAAAATAAATTTТАТСAATCC & & \\
\hline sitA02 & GGTGTAGCATAGGATCCCTC & BamHI & \\
\hline sitA03 & ATTTCTTGATCTTCGCCCGG & & \\
\hline sitA05 & GTTATATAGCACAAGCTATTTAT & & \\
\hline sitA06 & ATTAACCACACCATTGCGAG & & \\
\hline galF01 & TCAGCGGCTCGTTCCTTTG & & \\
\hline $\mathrm{cc02}$ & GGATCCTCTCATGTCTTACTTAACC & $\mathrm{BamHI}$ & \\
\hline For RT-qPCR & Sequence $\left(5^{\prime} \rightarrow 3^{\prime}\right)$ & TaqMan probes & Target \\
\hline RT11 & GGTAGGGGAGCGTTCTGTAA & 67 & $23 \mathrm{~S}$ rRNA \\
\hline RT12 & TCAGCATTCGCACTTCTGAT & & \\
\hline GT56 & ACCCCGCCAGCTTTAACTT & 3 & entC \\
\hline GT57 & TGTCCTTCTTTACGCAGCAG & & \\
\hline GT58 & CAACCTGAACAGCGATTTCC & 20 & fecA \\
\hline GT59 & TCGGCGCTCTCTTTAACAGT & & \\
\hline GT62 & CAGATGTCAGCGCAGATCC & 20 & $f e o B$ \\
\hline GT63 & CATAGGCCCGGCTGTAGA & & \\
\hline GT64 & AAAGAGATTGGCCTCGAGTTT & 20 & fерA \\
\hline GT59 & TCGGCGCTCTCTTTAACAGT & & \\
\hline GT66 & AATAAACAGCTCGTTTCGTTAAAAG & 160 & fep $B$ \\
\hline GT67 & GTATAGACCAGGGCGGTCAC & & \\
\hline GT68 & GTTTGGTCGTATCGCCTGAC & 3 & fhuA \\
\hline GT69 & GGAAGGTGAAGTCAGTTTTATCG & & \\
\hline GT72 & TGATGACCTACCTGCAGTACCA & 20 & hmuR \\
\hline GT73 & GAGCCGAGGTTCCAGGAG & & \\
\hline GT74 & CGGAGGAACATTCGTCAAA & 84 & iroB \\
\hline GT75 & TTCGGAATCTAAGCCTGGTG & & \\
\hline
\end{tabular}


Table 3. Cont.

\begin{tabular}{llll}
\hline & & & TaqMan probes \\
\hline For RT-qPCR & Sequence $\left(\mathbf{5}^{\prime} \rightarrow \mathbf{3}^{\prime}\right)$ & Target & iucA \\
\hline GT78 & TCTCCCGGCTATTGTTGATA & 67 & sitA \\
GT79 & GGAAGGTTCGCAACTGGT & 20 & \\
GT82 & GAAGATCCGTCAGACGATGG & & \\
\hline doi: 10.1371 /journal.pone.0107812.t003 & TAGTCGCGGGCAGATAG & &
\end{tabular}

LightCycler 1.5 Instrument using LightCycler TaqMan Master (Roche). Primers and probes were designed for selected target sequences using Universal ProbeLibrary Assay Design Center (Roche-applied science) and listed in Table 3. Data were analyzed using the real time PCR software of Roche LightCycler 1.5 Instrument. Relative gene expressions were quantified using the comparative threshold cycle $2^{-\Delta \Delta \mathrm{CT}}$ method with $23 \mathrm{~S}$ rRNA as the endogenous reference.

\section{CAS assay}

The CAS assay was performed according to the method described by Schwyn and Neilands [40]. Each of the bacterial strain was grown overnight in LB medium, and then $5 \mu \mathrm{l}$ of culture was added onto a CAS agar plate. After $24 \mathrm{~h}$ incubation at $37^{\circ} \mathrm{C}$, the effects of the bacterial siderophore production could be observed. Siderophore production was apparent as a halo around the colonies; the absence of a halo indicated the inability to produce siderophores.

\section{Statistical methods}

An unpaired t-test was used to determine the statistical significance and values of $P<0.01$ were considered significant. The results of CPS quantification, $\beta$-galactosidase activity, serum survival rate, and qRT-PCR analysis were performed in triplicate and independently repeated at least three times, and the mean activity and standard deviation are presented.

\section{References}

1. Podschun R, Ullmann U (1998) Klebsiella spp. as nosocomial pathogens: epidemiology, taxonomy, typing methods, and pathogenicity factors. Clin Microbiol Rev 11: 589-603.

2. Han SH (1995) Review of hepatic abscess from Klebsiella pneumoniae. An association with diabetes mellitus and septic endophthalmitis. West J Med 162: 220-224.

3. Lau YJ, Hu BS, Wu WL, Lin YH, Chang HY, et al. (2000) Identification of a major cluster of Klebsiella pneumoniae isolates from patients with liver abscess in Taiwan. J Clin Microbiol 38: 412-414.

4. Yang YS, Siu LK, Yeh KM, Fung CP, Huang SJ, et al. (2009) Recurrent Klebsiella pneumoniae liver abscess: clinical and microbiological characteristics. J Clin Microbiol 47: 3336-3339.

5. Sahly H, Podschun R, Oelschlaeger TA, Greiwe M, Parolis H, et al. (2000) Capsule impedes adhesion to and invasion of epithelial cells by Klebsiella pneumoniae. Infect Immun 68: 6744-6749.

6. Lin JC, Chang FY, Fung CP, Xu JZ, Cheng HP, et al. (2004) High prevalence of phagocytic-resistant capsular serotypes of Klebsiella pneumoniae in liver abscess. Microbes Infect 6: 1191-1198.

7. Boddicker JD, Anderson RA, Jagnow J, Clegg S (2006) Signature-tagged mutagenesis of Klebsiella pneumoniae to identify genes that influence biofilm formation on extracellular matrix material. Infect Immun 74: 4590-4597.

8. Moranta D, Regueiro V, March C, Llobet E, Margareto J, et al. (2010) Klebsiella pneumoniae capsule polysaccharide impedes the expression of betadefensins by airway epithelial cells. Infect Immun 78: 1135-1146.

9. Favre-Bonte S, Joly B, Forestier C (1999) Consequences of reduction of Klebsiella pneumoniae capsule expression on interactions of this bacterium with epithelial cells. Infect Immun 67: 554-561.

10. Lin CT, Wu CG, Chen YS, Lai YC, Chi C, et al. (2010) Fur regulation of the capsular polysaccharide biosynthesis and iron-acquisition systems in Klebsiella pneumoniae CG43. Microbiology 157: 419-429.

\section{Ethics statement}

For isolation of normal human serum from healthy volunteers, the procedure and the respective consent documents were approved by the Ethics Committee of the China Medical University Hospital, Taichung, Taiwan. All healthy volunteers provided written informed consent.

\section{Supporting Information}

Figure S1 Single-copy complementation of iscR but not iscR $R_{3 \mathrm{CA}}$ in the AP001 strain restores native production levels of CPS. CPS levels of the $K$. pneumoniae strains, as indicated, grown in LB broth were determined as described in Materials and Methods $\left({ }^{*} P<0.01\right)$.

(TIF)

\section{Methods S1 Supporting Materials and Methods. (DOCX)}

\section{Author Contributions}

Conceived and designed the experiments: CTL GKW YCG CGW THL. Performed the experiments: CTL GKW YCG CGW THL. Analyzed the data: CTL CCW TRJ. Contributed reagents/materials/analysis tools: CTL GKW YCG THL. Wrote the paper: CTL CGW.

11. Huang SH, Wang CK, Peng HL, Wu CC, Chen YT, et al. (2012) Role of the small RNA RyhB in the Fur regulon in mediating the capsular polysaccharide biosynthesis and iron acquisition systems in Klebsiella pneumoniae. BMC Microbiol 12: 148

12. Andrews SC, Robinson AK, Rodriguez-Quinones F (2003) Bacterial iron homeostasis. FEMS Microbiol Rev 27: 215-237.

13. Outten FW, Djaman O, Storz G (2004) A suf operon requirement for Fe-S cluster assembly during iron starvation in Escherichia coli. Mol Microbiol 52: 861-872.

14. Johnson DC, Dean DR, Smith AD, Johnson MK (2005) Structure, function, and formation of biological iron-sulfur clusters. Annu Rev Biochem 74: 247-281.

15. Ayala-Castro C, Saini A, Outten FW (2008) Fe-S cluster assembly pathways in bacteria. Microbiol Mol Biol Rev 72: 110-125.

16. Giel JL, Nesbit AD, Mettert EL, Fleischhacker AS, Wanta BT, et al. (2013) Regulation of iron-sulphur cluster homeostasis through transcriptional control of the Isc pathway by [2Fe-2S]-IscR in Escherichia coli. Mol Microbiol 87: 478492.

17. Pullan ST, Gidley MD, Jones RA, Barrett J, Stevanin TM, et al. (2007) Nitric oxide in chemostat-cultured Escherichia coli is sensed by Fnr and other global regulators: unaltered methionine biosynthesis indicates lack of $\mathrm{S}$ nitrosation. J Bacteriol 189: 1845-1855.

18. Yeo WS, Lee JH, Lee KC, Roe JH (2006) IscR acts as an activator in response to oxidative stress for the suf operon encoding $\mathrm{Fe}-\mathrm{S}$ assembly proteins. Mol Microbiol 61: 206-218.

19. Giel JL, Rodionov D, Liu M, Blattner FR, Kiley PJ (2006) IscR-dependent gene expression links iron-sulphur cluster assembly to the control of O2-regulated genes in Escherichia coli. Mol Microbiol 60: 1058-1075.

20. Schwartz CJ, Giel JL, Patschkowski T, Luther C, Ruzicka FJ, et al. (2001) IscR, an $\mathrm{Fe}-\mathrm{S}$ cluster-containing transcription factor, represses expression of 
Escherichia coli genes encoding Fe-S cluster assembly proteins. Proc Natl Acad Sci U S A 98: 14895-14900.

21. Nesbit AD, Giel JL, Rose JC, Kiley PJ (2009) Sequence-specific binding to a subset of IscR-regulated promoters does not require IscR Fe-S cluster ligation. J Mol Biol 387: 28-41.

22. Arakawa Y, Wacharotayankun R, Nagatsuka T, Ito H, Kato N, et al. (1995) Genomic organization of the Klebsiella pneumoniae cps region responsible for serotype K2 capsular polysaccharide synthesis in the virulent strain Chedid. J Bacteriol 177: 1788-1796.

23. Lin CT, Huang TY, Liang WC, Peng HL (2006) Homologous response regulators $\mathrm{KvgA}$, KvhA and KvhR regulate the synthesis of capsular polysaccharide in Klebsiella pneumoniae CG43 in a coordinated manner. J Biochem (Tokyo) 140: 429-438.

24. Regueiro V, Campos MA, Pons J, Alberti S, Bengoechea JA (2006) The uptake of a Klebsiella pneumoniae capsule polysaccharide mutant triggers an inflammatory response by human airway epithelial cells. Microbiology 152: $555-566$.

25. Lin CT, Chen YC, Jinn TR, Wu CC, Hong YM, et al. (2013) Role of the cAMP-Dependent Carbon Catabolite Repression in Capsular Polysaccharide Biosynthesis in Klebsiella pneumoniae. PLoS One 8: e54430.

26. da Silva Neto JF, Braz VS, Italiani VC, Marques MV (2009) Fur controls iron homeostasis and oxidative stress defense in the oligotrophic alpha-proteobacterium Caulobacter crescentus. Nucleic Acids Res 37: 4812-4825.

27. Kiley PJ, Beinert $\mathrm{H}$ (2003) The role of Fe-S proteins in sensing and regulation in bacteria. Curr Opin Microbiol 6: 181-185.

28. Griffith KL, Wolf RE Jr (2001) Systematic mutagenesis of the DNA binding sites for SoxS in the Escherichia coli zwf and $f p r$ promoters: identifying nucleotides required for DNA binding and transcription activation. Mol Microbiol 40: 1141-1154.

29. Rajagopalan S, Teter SJ, Zwart PH, Brennan RG, Phillips KJ, et al. (2013) Studies of IscR reveal a unique mechanism for metal-dependent regulation of DNA binding specificity. Nat Struct Mol Biol 20: 740-747.

30. Lim JG, Choi SH (2013) IscR is a global regulator essential for pathogenesis of Vibrio vulnificus and induced by host cells. Infect Immun 82: 569-578.
31. Wu CC, Lin CT, Cheng WY, Huang CJ, Wang ZC, et al. (2012) Fur-dependent MrkHI regulation of type 3 fimbriae in Klebsiella pneumoniae CG43. Microbiology 158: 1045-1056.

32. Wilks A, Burkhard KA (2007) Heme and virulence: how bacterial pathogens regulate, transport and utilize heme. Nat Prod Rep 24: 511-522.

33. Miller RA, Britigan BE (1997) Role of oxidants in microbial pathophysiology. Clin Microbiol Rev 10: 1-18.

34. Py B, Moreau PL, Barras F (2011) Fe-S clusters, fragile sentinels of the cell. Curr Opin Microbiol 14: 218-223.

35. Lai YC, Peng HL, Chang HY (2003) RmpA2, an activator of capsule biosynthesis in Klebsiella pneumoniae CG43, regulates K2 cps gene expression at the transcriptional level. J Bacteriol 185: 788-800.

36. Skorupski K, Taylor RK (1996) Positive selection vectors for allelic exchange. Gene 169: 47-52.

37. Miller VL, Mekalanos JJ (1988) A novel suicide vector and its use in construction of insertion mutations: osmoregulation of outer membrane proteins and virulence determinants in Vibrio cholerae requires toxR. J Bacteriol 170: 25752583.

38. Domenico P, Schwartz S, Cunha BA (1989) Reduction of capsular polysaccharide production in Klebsiella pneumoniae by sodium salicylate. Infect Immun 57: 3778-3782.

39. Blumenkrantz N, Asboe-Hansen G (1973) New method for quantitative determination of uronic acids. Anal Biochem 54: 484-489.

40. Schwyn B, Neilands JB (1987) Universal chemical assay for the detection and determination of siderophores. Anal Biochem 160: 47-56.

41. Lai YC, Peng HL, Chang HY (2001) Identification of genes induced in vivo during Klebsiella pneumoniae CG43 infection. Infect Immun 69: 7140-7145.

42. Cheng HY, Chen YS, Wu CY, Chang HY, Lai YC, et al. (2010) RmpA regulation of capsular polysaccharide biosynthesis in Klebsiella pneumoniae CG43. J Bacteriol 192: 3144-3158.

43. Hanahan D (1983) Studies on transformation of Escherichia coli with plasmids. J Mol Biol 166: 557-580. 